\title{
The Cauchy problem for the generalized Degasperis-Procesi equation
}

\author{
Fei Zuo*, Changan Tian and Hongjun Wang
}

${ }^{*}$ Correspondence: feizuo2006@163.com

College of Mathematics and Information Science, Henan Normal University, Xinxiang, Henan 453007, P.R. China

\begin{abstract}
In this paper, we investigate the Cauchy problem for the generalized Degasperis-Procesi equation in a Besov space. Firstly, we prove that the generalized Degasperis-Procesi equation is locally well posed in $B_{p, r}^{s}$ with $s>1+\frac{1}{p}$ (or $s \geq 1+\frac{1}{p}$ if $r=1$ with $p \in[1,+\infty)$ ). Secondly, we prove that the generalized Degasperis-Procesi equation possesses the peaked solitary wave which is the weak solution to the generalized Degasperis-Procesi equation. Thirdly, we prove that the data-to-solution map for the generalized Degasperis-Procesi equation is not uniformly continuous in $B_{2, \infty}^{3 / 2}$. Fourthly, we prove that the data-to-solution map for the generalized Degasperis-Procesi equation is not uniformly continuous in $H^{5}(\mathbf{R})$ with $s<3 / 2$. Finally, we give a blow-up criterion.

MSC: $35 \mathrm{G} 25 ; 35 \mathrm{~L} 05$
\end{abstract}

Keywords: Cauchy problem; generalized Degasperis-Procesi equation; weak solution; blow-up criterion

\section{Introduction}

In this paper, we consider the Cauchy problem for the following generalized DegasperisProcesi equation:

$$
\begin{aligned}
& u_{t}-u_{t x x}+u^{k} u_{x}-\left(u^{k} u_{x}\right)_{x x}+Q\left[u^{k+1}\right]_{x}=0, \\
& u(x, 0)=u_{0}(x),
\end{aligned}
$$

where $Q \in \mathbf{R}$ is a constant. When $k=1$ and $Q=\frac{3}{2}$, (1.1) reduces to the Degasperis-Procesi equation

$$
u_{t}-u_{t x x}+4 u u_{x}=3 u_{x} u_{x x}+u u_{x x x}
$$

Equation (1.3) possesses the Lax pair and bi-Hamiltonian structures and infinite many conservation laws [1]. The Degasperis-Procesi equation [2] possesses peaked solitons which are stable [3] and shock peakons of the form $u(x, t)=-\frac{1}{t+k} \operatorname{sign}(x) e^{-|x|}, k>0$. The Degasperis-Procesi equation possesses the global weak solution and blow-up structure [4-6]. Constantin and Lannes studied the relevance between the Camassa-Holm equation and the Degasperis-Procesi equation [7]. The Degasperis-Procesi equation possesses the infinite propagation speed [8]. The Degasperis-Procesi equation possesses multi-peakon solutions [9] and multisoliton [10]. Himonas and his co-authors [11, 12] proved that the

\section{盟 Springer}

๑2013 Zuo et al.; licensee Springer. This is an Open Access article distributed under the terms of the Creative Commons Attribution License (http://creativecommons.org/licenses/by/2.0), which permits unrestricted use, distribution, and reproduction in any medium, provided the original work is properly cited. 
data-to-solution for the Camassa-Holm equation, the Degasperis-Procesi equation is not uniformly continuous in $H^{s}(\mathbf{R})$ with $s>3 / 2$, respectively. Himonas et al. proved the nonuniform continuity in $H^{1}$ of the solution map of the $\mathrm{CH}$ equation [13]. Recently, Gui and Liu [14] studied the Cauchy problem for the Degasperis-Procesi equation in Besov spaces. Yan et al. [15] studied the Cauchy problem for the Novikov equation in Besov spaces.

Let $P(D)=-\partial_{x}\left(1-\partial_{x}^{2}\right)^{-1}$ and $p(x)=\frac{1}{2} e^{-|x|}, x \in \mathbf{R}$. By using the identity $\left(1-\partial_{x}^{2}\right)^{-1} f=p * f$ for $f \in L^{2}$, we can rewrite (1.1)-(1.2) as follows:

$$
\begin{aligned}
& u_{t}+u^{k} u_{x}=Q P(D)\left[u^{k+1}\right], \\
& u(x, 0)=u_{0}(x) .
\end{aligned}
$$

In this paper, motivated by [14], we study the Cauchy problem for (1.4) in Besov spaces. Firstly, we use the standard iterative method to prove that the generalized DegasperisProcesi equation is locally well posed in $B_{p, r}^{s}$ with $s>1+\frac{1}{p}$ (or $s \geq 1+\frac{1}{p}$ if $r=1$ with $p \in$ $[1,+\infty)$ ). Secondly, we prove that the generalized Degasperis-Procesi equation possesses the peaked solitary wave which is the weak solution to the generalized Degasperis-Procesi equation. Thirdly, we prove that the data-to-solution map for the generalized DegasperisProcesi equation is not uniformly continuous in $B_{2, \infty}^{3 / 2}$. Fourthly, we prove that the data-tosolution map for the generalized Degasperis-Procesi equation is not uniformly continuous in $H^{s}(\mathbf{R})$ with $s<3 / 2$. Finally, we give a blow-up criterion.

Notice that the structure of (1.4) is more complicated than that of the DegasperisProcesi equation. Thus, to prove that the sequence of smooth solutions $\left(u^{(n)}\right)_{n \in \mathbf{N}}$ is uniformly bounded in $C\left([0, T] ; B_{p, r}^{s}\right) \cap C^{1}\left([0, T] ; B_{p, r}^{s-1}\right)$ with $s>1+\frac{1}{p}$ (or $s \geq 1+\frac{1}{p}$ if $r=1$ with $p \in[1,+\infty))$, we choose that

$$
\left\|u^{(n)}\right\|_{B_{p, r}^{s}} \leq \frac{\left\|u_{0}\right\|_{B_{p, r}^{s}}}{\left(1-2 k C\left\|u_{0}\right\|_{B_{p, r}^{s}}^{k} t\right)^{1 / k}}, \quad t \in[0, T] .
$$

In proving Theorem 1.5, we explain why we choose (1.6). It is worthy of pointing out that we use Fatou's lemma and the upper limit as well as Gronwall's inequality to prove that $\left(u^{(n)}\right)_{n \in \mathbf{N}}$ is a Cauchy sequence in $C\left([0, T] ; B_{p, r}^{s-1}\right)$ with $s>1+\frac{1}{p}$ (or $s \geq 1+\frac{1}{p}$ if $r=1$ with $p \in[1,+\infty))$.

To introduce the main results, we define

$$
E_{p, r}^{s}(T)=C\left([0, T] ; B_{p, r}^{s}\right) \cap C^{1}\left([0, T] ; B_{p, r}^{s-1}\right) .
$$

The main results of this paper are as follows.

Theorem 1.1 Let $u_{0}(x) \in B_{p, r}^{s}$ with $s>1+\frac{1}{p}$ (or $s \geq 1+\frac{1}{p}$ if $r=1$ with $p \in[1,+\infty)$ ). Problem (1.4)-(1.5) is locally well posed. Moreover,

$$
\|u(t)\|_{B_{p, r}^{s}} \leq \frac{\left\|u_{0}\right\|_{B_{p, r}^{s}}}{\left(1-2 k C\left\|u_{0}\right\|_{B_{p, r}^{s}}^{k} t\right)^{1 / k}}, \quad t \in[0, T] .
$$


A function $u:[0, T) \times \mathbf{R}$ is called a weak solution to (1.1) (or (1.4)) if $u$ belongs to $L_{\mathrm{loc}}^{\infty}\left([0, T) ; H^{1}\right)$ and satisfies the following identity:

$$
\begin{aligned}
& \int_{0}^{T} \int_{\mathbf{R}}\left[u \phi_{t}+\frac{1}{k+1} u^{k+1} \phi_{x}+p *\left[\frac{k^{2}+2 k}{k+1} u^{k+1}\right] \phi_{x}\right] d x d t \\
& \quad+\int_{\mathbf{R}} u(x, 0) \phi(x, 0) d x=0,
\end{aligned}
$$

where $p(x)=\frac{1}{2} e^{-|x|}$, for any smooth test function $\phi(x, t) \in C_{c}^{\infty}([0, T) \times \mathbf{R})$. If $u$ is a weak solution on $[0, T)$ for every $T>0$, then it is called a global weak solution.

Theorem 1.2 When $Q=\frac{k(k+2)}{k+1}$ in (1.4), $u_{c}(x, t)=c^{1 / k} e^{-|x-c t|}$ with $c>0$ is a weak solution of (1.4) in the sense of (1.8).

Theorem 1.3 When $Q=\frac{k(k+2)}{k+1}$ in (1.4), the data-to-solution map for the generalized Degasperis-Procesi equation is not uniformly continuous in $B_{2, \infty}^{3 / 2}$. More precisely, there exists a global solution $u \in L^{\infty}\left(\mathbf{R}^{+} ; B_{2, \infty}^{3 / 2}\right)$ to the Cauchy problem for (1.4) such that for any $T>0$ and $\epsilon>0$, there exists a solution $v \in L^{\infty}\left(0, T ; B_{2, \infty}^{3 / 2}\right)$ with

$$
\|v(0)-u(0)\|_{B_{2, \infty}^{3 / 2}} \leq \epsilon, \quad\|v(t)-u(t)\|_{L^{\infty}\left(0, T ; B_{2, \infty}^{3 / 2}\right)} \geq 1
$$

Theorem 1.4 When $Q=\frac{k(k+2)}{k+1}$ in (1.4), the data-to-solution map for the generalized Degasperis-Procesi equation is not uniformly continuous in $H^{s}(\mathbf{R})$ with $s<3 / 2$.

Theorem 1.5 Assume that $T^{\star}$ is the maximal time of existence of the solution to problem (1.4)-(1.5). If $T^{\star}<\infty$, then

$$
\int_{0}^{T^{\star}}\left\|u_{x}\right\|_{L^{\infty}}^{k} d \tau=+\infty
$$

Moreover, $T^{\star} \geq \frac{1}{C k\left\|u_{0}\right\|_{B_{p, r}^{s}}^{k}}$.

The remainder of this paper is organized as follows. In Section 2, we give some preliminaries. In Section 3, we prove Theorem 1.1. In Section 4, we prove Theorem 1.2. In Section 5, we prove Theorem 1.3. In Section 6, we prove Theorem 1.4. In Section 7, we prove Theorem 1.5 .

\section{Preliminaries}

In this section, we give Lemmas 2.1-2.4. The proof of Lemmas 2.1-2.4 can be seen in [16-21].

Lemma 2.1 (Littlewood-Paley decomposition) Let $B=\left\{\xi \in \mathbf{R}^{n},|\xi| \leq \frac{4}{3}\right\}$ and $C=\{\xi \in$ $\left.\mathbf{R}^{n}, \frac{3}{4} \leq|\xi| \leq \frac{8}{3}\right\}$. There exists a couple of smooth radial functions $(\chi, \phi) \in\left(C_{c}^{\infty}(B), C_{c}^{\infty}(C)\right)$ such that

$$
\forall \xi \in \mathbf{R}^{n}, \quad \chi(\xi)+\sum_{q \in \mathbf{N}} \phi\left(2^{-q} \xi\right)=1
$$


and

$$
\begin{aligned}
& \operatorname{Supp} \phi\left(2^{-q} \cdot\right) \cap \operatorname{Supp} \phi\left(2^{-q^{\prime}} .\right)=\emptyset \quad \text { if }\left|q-q^{\prime}\right| \geq 2, \\
& \operatorname{Supp} \chi(\cdot) \cap \operatorname{Supp} \phi\left(2^{-q} \cdot\right)=\emptyset \quad \text { if }|q| \geq 1
\end{aligned}
$$

and

$$
\frac{1}{3} \leq \chi(\xi)^{2}+\sum_{q \geq 0} \phi\left(2^{-q} \xi\right)^{2} \leq 1, \quad \forall \xi \in \mathbf{R}^{n}
$$

Then, for $u \in \mathscr{S}^{\prime}(\mathbf{R})$, the nonhomogeneous dyadic blocks are defined as follows:

$$
\begin{aligned}
& \Delta_{q} u=0 \quad \text { if } q \leq-2, \\
& \Delta_{-1} u=\chi(D) u=\mathscr{F}_{x}^{-1} \chi \mathscr{F}_{x} u, \\
& \Delta_{q} u=\phi\left(2^{-q} D\right)=\mathscr{F}_{x}^{-1} \phi\left(2^{-q} \xi\right) \mathscr{F}_{x} u \quad \text { if } q \geq 0 .
\end{aligned}
$$

Thus we obtain

$$
u=\sum_{q \in \mathbf{Z}} \Delta_{q} u \quad \text { in } \mathscr{S}^{\prime}(\mathbf{R})
$$

and the low frequency cut-off $S_{q}$ is defined by

$$
S_{q} u=\sum_{p=-1}^{q-1} \Delta_{p} u=\chi\left(2^{-q} D\right) u=\mathscr{F}_{x}^{-1} \chi\left(2^{-q} \xi\right) \mathscr{F}_{x} u, \quad \forall q \in \mathbf{N}
$$

as well as

$$
\begin{aligned}
& \Delta_{p} \Delta_{q} u \equiv 0 \quad \text { if }|p-q| \geq 2, \\
& \Delta_{q}\left(S_{p-1} u \Delta_{p} v\right) \equiv 0 \quad \text { if }|p-q| \geq 5, \forall u, v \in \mathscr{S}^{\prime}(\mathbf{R}), \\
& \left\|\Delta_{p} u\right\|_{L^{p}} \leq C\|u\|_{L^{p}}, \\
& \left\|S_{q} u\right\|_{L^{p}} \leq C\|u\|_{L^{p}}, \quad \forall 1 \leq p \leq+\infty,
\end{aligned}
$$

where $C$ is a positive constant independent of $q$.

Definition (Besov spaces) Let $s \in \mathbf{R}$ and $1 \leq p \leq+\infty$. The nonhomogeneous Besov space $B_{p, r}^{s}\left(\mathbf{R}^{n}\right)$ is defined by

$$
\begin{aligned}
& B_{p, r}^{s}\left(\mathbf{R}^{n}\right) \\
& \quad=\left\{f \in \mathscr{S}^{\prime}\left(\mathbf{R}^{n}\right):\|f\|_{B_{p, r}^{s}}=\left\|2^{q s} \Delta_{q} f\right\|_{l^{r}\left(L^{p}\right)}=\left\|\left(2^{q s}\left\|\Delta_{q} f\right\|_{L^{p}}\right)_{q \geq-1}\right\|_{l^{r}}<\infty\right\} .
\end{aligned}
$$

In particular, if $s=\infty$, then $B_{p, r}^{s}=\bigcap_{s \in \mathbf{R}} B_{p, r}^{s}$.

Lemma 2.2 Let $s \in \mathbf{R}, 1 \leq p, r, p_{j}, r_{j} \leq \infty, j=1,2$, then: 
(1) $B_{p, r}^{s}$ is a Banach space and is continuously embedded in $\mathscr{S}^{\prime}\left(\mathbf{R}^{n}\right)$.

(2) $B_{p_{1}, r_{1}}^{s_{1}} \hookrightarrow B_{p_{2}, r_{2}}^{s_{2}}$, if $p_{1} \leq p_{2}$ and $r_{1} \leq r_{2}$ and $s_{2}=s_{1}-n\left(\frac{1}{p_{1}}-\frac{1}{p_{2}}\right)$

$$
B_{p, r_{2}}^{s_{1}} \hookrightarrow B_{p, r_{1}}^{s_{2}} \quad \text { locally compact if } s_{2}<s_{1} .
$$

(3) $\forall s>0, B_{p, r}^{s} \cap L^{\infty}$ is a Banach algebra. $B_{p, r}^{s}$ is a Banach algebra iff $B_{p, r}^{s} \hookrightarrow L^{\infty}$ and iff $s>\frac{1}{p}$ or $\left(s \geq \frac{1}{p}\right.$ and $\left.r=1\right)$.

(4) (i) For $s>0$,

$$
\|f g\|_{B_{p, r}^{s}} \leq C\left(\|f\|_{B_{p, r}^{s}}\|g\|_{L^{\infty}}+\|f\|_{L^{\infty}}\|g\|_{B_{p, r}^{s}}\right), \quad \forall f, g \in B_{p, r}^{s} \cap L^{\infty} .
$$

(ii) $\forall s_{1} \leq \frac{1}{p}<s_{2}\left(s_{2} \geq \frac{1}{p}\right.$ if $\left.r=1\right)$ and $s_{1}+s_{2}>0$,

$$
\|f g\|_{B_{p, r}^{s_{1}}} \leq C\|f\|_{B_{p, r}^{s_{1}}}\|g\|_{B_{p, r}^{s_{2}}}, \quad \forall f \in B_{p, r}^{s_{1}}, g \in B_{p, r}^{s_{2}} .
$$

(5) $\forall \theta \in[0,1]$ and $s=\theta s_{1}+(1-\theta) s_{2}$,

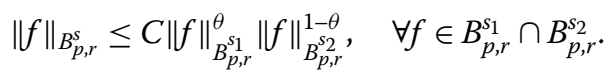

(6) If $\left(u_{n}\right)_{n \in \mathbf{N}}$ is bounded in $B_{p, r}^{s}$ and $u_{n} \rightarrow u$ in $\mathscr{S}^{\prime}\left(\mathbf{R}^{n}\right)$, then $u \in B_{p, r}^{s}$ and

$$
\|u\|_{B_{p, r}^{s}} \leq \liminf _{n \rightarrow \infty}\left\|u_{n}\right\|_{B_{p, r}^{s}}
$$

(7) Let $m \in \mathbf{R}$ and $\Psi$ be an $S^{m}$-multiplier. Then the operator $\Psi(D)$ is continuous from $B_{p, r}^{s}$ into $B_{p, r}^{s-m}$. In particular, $-\partial_{x}\left(1-\partial_{x}^{2}\right)^{-1}$ is continuous from $B_{p, r}^{s}$ into $B_{p, r}^{s-1}$.

Lemma 2.3 (A priori estimates in Besov spaces) Let $1 \leq p, r \leq \infty$ and $s>-\min \left\{\frac{1}{p}, 1-\frac{1}{p}\right\}$. Assume that $f_{0} \in B_{p, r}^{s}, F \in L^{1}\left(0, T ; B_{p, r}^{s}\right)$ and that $\partial_{x} v$ belongs to $L^{1}\left(0, T ; B_{p, r}^{s-1}\right)$ if $s>1+\frac{1}{p}$ or to $L^{1}\left(0, T ; B_{p, r}^{1 / p} \cap L^{\infty}\right)$ otherwise. If $\in L^{\infty}\left(0, T ; B_{p, r}^{s}\right) \cap C\left([0, T] ; \mathscr{S}^{\prime}(\mathbf{R})\right)$ solves the following 1-D linear transport equation:

$$
\begin{aligned}
& f_{t}+v f_{x}=F, \\
& f(x, 0)=f_{0},
\end{aligned}
$$

then there exists a constant $C$ depending only on $s, p, r$ such that the following statements hold:

(1) If $r=1$ or $s \neq 1+\frac{1}{p}$, then

$$
\|f\|_{B_{p, r}^{s}} \leq\left\|f_{0}\right\|_{B_{p, r}^{s}}+\int_{0}^{t}\|F(\tau)\|_{B_{p, r}^{s}} d \tau+C \int_{0}^{t} V^{\prime}(\tau)\|f(\tau)\|_{B_{p, r}^{s}} d \tau
$$

or

$$
\|f\|_{B_{p, r}^{s}} \leq e^{C V(t)}\left(\left\|f_{0}\right\|_{B_{p, r}^{s}}+\int_{0}^{t} e^{-C V(\tau)}\|F(\tau)\|_{B_{p, r}^{s}} d \tau\right)
$$

with $V(t)=\int_{0}^{t}\left\|v_{x}(\tau)\right\|_{B_{p, r}^{1 / p} \cap L^{\infty}} d \tau$ if $s<1+\frac{1}{p}$ and $V(t)=\int_{0}^{t}\left\|v_{x}(\tau)\right\|_{B_{p, r}^{s-1}} d \tau$ else. 
(2) If $s \leq 1+\frac{1}{p}, f_{0}^{\prime} \in L^{\infty}$ and $f_{x} \in L^{\infty}((0, T) \times \mathbf{R})$ and $F_{x} \in L^{1}\left(0, T ; L^{\infty}\right)$, then

$$
\begin{aligned}
& \|f(t)\|_{B_{p, r}^{s}}+\left\|f_{x}(t)\right\|_{L^{\infty}} \\
& \quad \leq e^{C V(t)}\left(\left\|f_{0}\right\|_{B_{p, r}^{s}}+\left\|f_{0}^{\prime}\right\|_{L^{\infty}}+\int_{0}^{t} e^{-C V(\tau)}\left[\|F(\tau)\|_{B_{p, r}^{s}}+\left\|F_{x}(\tau)\right\|_{L^{\infty}}\right] d \tau\right)
\end{aligned}
$$

with

$$
V(t)=\int_{0}^{t}\left\|\partial_{x} \nu(\tau)\right\|_{B_{p, r}^{1 / p} \cap L^{\infty}} d \tau .
$$

(3) Iff $=v$, then for all $s>0$, (1) holds true when $V(t)=\int_{0}^{t}\left\|v_{x}(\tau)\right\|_{L^{\infty}} d \tau$.

(4) If $r<\infty$, then $f \in C\left([0, T] ; B_{p, r}^{s}\right)$. If $r=\infty$, then $f \in C\left([0, T] ; B_{p, 1}^{s^{\prime}}\right)$ for all $s^{\prime}<s$.

Lemma 2.4 (Existence and uniqueness) Let $p, r, s, f_{0}$ and $F$ be as in the statement of Lemma 2.3. Assume that $v \in L^{\rho}\left(0, T ; B_{\infty, \infty}^{-M}\right)$ for some $\rho>1$ and $M>0$ and $v_{x} \in L^{1}\left(0, T ; B_{p, r}^{s-1}\right)$ if $s>1+\frac{1}{p}$ or $s=1+\frac{1}{p}$ and $r=1$ and $v_{x} \in L^{1}\left(0, T ; B_{p, \infty}^{1 / p} \cap L^{\infty}\right)$ if $s<1+\frac{1}{p}$. Then problem (2.1)(2.2) has a unique solution $f \in L^{\infty}\left(0, T ; B_{p, r}^{s}\right) \cap\left(\bigcap_{s^{\prime}<s} C\left([0, T] ; B_{p, 1}^{s^{\prime}}\right)\right)$ and the inequalities of Lemma 2.3 can hold true. Moreover, if $r<\infty$, then $f \in C\left([0, T] ; B_{p, r}^{s}\right)$.

\section{Proof of Theorem 1.1}

In this section, we complete the proof of Theorem 1.1 and suppose that $s>1+\frac{1}{p}$ (or $s \geq 1+\frac{1}{p}$ if $r=1$ with $p \in[1,+\infty))$.

\section{First step: approximate solution}

By using the standard iterative process, we construct a sequence of smooth solutions $\left(u^{(n)}\right)_{n \in \mathbf{N}} \in C\left(\mathbf{R}^{+} ; B_{p, r}^{\infty}\right)$. Assume that $u^{(0)}:=0$, by induction we define a sequence of smooth functions $\left(u^{(n)}\right)_{n \in \mathbf{N}}$ by solving the following linear transport equation:

$$
\begin{aligned}
& u_{t}^{(n+1)}+\left[u^{(n)}\right]^{k} u_{x}^{(n+1)}=Q P(D)\left[\left(u^{(n)}\right)^{k+1}\right], \\
& u^{(n+1)}(x, 0)=u_{0}^{(n+1)}(x)=S_{n+1} u_{0}(x) .
\end{aligned}
$$

By using the fact that $S_{n+1} u_{0}$ belong to $B_{p, r}^{\infty}$, from Lemma 2.4, for all $n \in \mathbf{N}$, we can show by induction that problem (3.1)-(3.2) has a global solution $\left(u^{(n)}\right)_{n \in \mathbf{N}} \in C\left(\mathbf{R}^{+}, B_{p, r}^{\infty}\right)$.

\section{Second step: uniform bounds}

For $s>1+\frac{1}{p}$ (or $s \geq 1+\frac{1}{p}$ if $r=1$ with $\left.p \in[1,+\infty)\right)$ and $n \in \mathbf{N}$, we prove that

$$
\begin{aligned}
& \left\|u^{(n+1)}\right\|_{B_{p, r}^{s}} \\
& \quad \leq e^{C U^{n}(t)}\left(\left\|u_{0}\right\|_{B_{p, r}^{s}}+C \int_{0}^{t} e^{-C U^{n}(\tau)}\left\|u^{(n)}\right\|_{B_{p, r}^{s}}^{k+1} d \tau\right),
\end{aligned}
$$

with $U^{n}=\int_{0}^{t}\left\|u^{(n)}\right\|_{B_{p, r}^{s}}^{k} d \tau$. 
From (2.3) of Lemma 2.3 and (3.1), we derive that

$$
\begin{aligned}
\left\|u^{(n+1)}(t)\right\|_{B_{p, r}^{s} \leq} \leq & e^{C \int_{0}^{t}\left\|u_{x}^{(n)}\left(t^{\prime}\right)\right\|_{B_{p, r}^{s-1}} d t^{\prime}}\left\|u_{0}\right\|_{B_{p, r}^{s}} \\
& +C \int_{0}^{t} e^{C \int_{\tau}^{t}\left\|u_{x}^{(n)}\left(t^{\prime}\right)\right\|_{B_{p, r}^{s-1}} d t^{\prime}}\left\|F\left(u^{(n)}\right)\right\|_{B_{p, r}^{s}} d \tau
\end{aligned}
$$

where

$$
F\left(u^{(n)}\right)=P(D)\left[\left(u^{(n)}\right)^{k+1}\right]
$$

By using the $S^{-1}$ multiplier property of $P(D)$ and the fact $B_{p, r}^{s}$ with $s>1+\frac{1}{p}$ (or $s \geq 1+\frac{1}{p}$ if $r=1$ with $p \in[1,+\infty))$ is a Banach algebra, we have

$$
\begin{aligned}
\left\|F\left(u^{(n)}\right)\right\|_{B_{p, r}^{s-1}} & \leq C\left\|P(D)\left[\left(u^{(n)}\right)^{k+1}\right]\left(t^{\prime}\right)\right\|_{B_{p, r}^{s}} \\
& \leq C\left\|\left(u^{(n)}\right)^{k+1}\left(t^{\prime}\right)\right\|_{B_{p, r}^{s-1}} \leq C\left\|u^{(n)}\left(t^{\prime}\right)\right\|_{B_{p, r}^{s}}^{k+1} .
\end{aligned}
$$

Inserting (3.6) into (3.4) yields (3.3).

Let us fix $T>0$ such that

$$
T \leq \frac{1}{4 k C\left\|u_{0}\right\|_{B_{p, r}^{s}}^{k}}
$$

and suppose that

$$
\left\|u^{(n)}(t)\right\|_{B_{p, r}^{s}} \leq \frac{\left\|u_{0}\right\|_{B_{p, r}^{s}}}{\left(1-2 k C\left\|u_{0}\right\|_{B_{p, r}^{s}}^{k} t\right)^{1 / k}}, \quad t \in[0, T] .
$$

Since $U^{n}(t)=\int_{0}^{t}\left\|u^{(n)}\right\|_{B_{p, r}^{s}}^{k} d \tau$, by using (3.8), we have

$$
\begin{aligned}
e^{C\left(U^{n}(t)-U^{n}(\tau)\right)} & =e^{C \int_{\tau}^{t}\left\|u^{(n)}\left(t^{\prime}\right)\right\|_{B_{p, r}^{s}}^{k} d t^{\prime}} \leq e^{-\frac{1}{2 k} \int_{\tau}^{t} \frac{d\left(1-2 k C\left\|u_{0}\right\|_{B_{p, r}^{s}}^{k} t^{\prime}\right)}{\left(1-2 k C\left\|u_{0}\right\|_{B_{p, r}^{s}}^{k} t^{\prime}\right)}} \\
& =\left(\frac{1-2 k C\left\|u_{0}\right\|_{B_{p, r}^{s}}^{k} \tau}{1-2 k C\left\|u_{0}\right\|_{B_{p, r}^{s}}^{k} t}\right)^{\frac{1}{2 k}}
\end{aligned}
$$

When $\tau=0$ in (3.9), we have

$$
e^{C U^{n}(t)} \leq\left(\frac{1}{1-2 k C\left\|u_{0}\right\|_{B_{p, r}^{s}}^{k} t}\right)^{\frac{1}{2 k}} .
$$

Inserting (3.9), (3.10) into (3.3) yields

$$
\begin{aligned}
\left\|u^{(n+1)}(t)\right\|_{B_{p, r}^{s}} & \leq \frac{\left\|u_{0}\right\|_{B_{p, r}^{s}}}{\left(1-2 k C\left\|u_{0}\right\|_{B_{p, r}^{s}}^{k} t\right)^{1 / 2 k}}\left[1-\frac{1}{2 k} \int_{0}^{t} \frac{d\left(1-2 k C\left\|u_{0}\right\|_{B_{p, r}^{s}}^{k} t\right)}{\left(1-2 k C\left\|u_{0}\right\|_{B_{p, r}^{s}}^{k} t\right)^{1+\frac{1}{2 k}}}\right] \\
& \leq \frac{\left\|u_{0}\right\|_{B_{p, r}^{s}}^{k}}{\left(1-2 k C\left\|u_{0}\right\|_{B_{p, r}^{s}}^{k} t\right)^{1 / k}} .
\end{aligned}
$$


Thus, $\left(u^{(n)}\right)_{n \in \mathbf{N}}$ is uniformly bounded in $C\left([0, T] ; B_{p, r}^{s}\right)$. By using (3.9) and the fact that $B_{p, r}^{s}$ is a Banach algebra and (3.5) and using the $S^{-1}$-multiplier property of $P(D)$, we have that

$$
\left\|\left[u^{(n)}\right]^{k} u_{x}^{(n+1)}\right\|_{B_{p, r}^{s}} \leq C\left\|u^{(n)}\right\|_{B_{p, r}^{s}}^{k}\left\|u^{(n+1)}\right\|_{B_{p, r}^{s}} \leq \frac{C\left\|u_{0}\right\|_{B_{p, r}^{s}}^{k+1}}{\left(1-2 C\left\|u_{0}\right\|_{B_{p, r}^{s}}^{k} t\right)^{\frac{k+1}{k}}}
$$

and

$$
\begin{aligned}
\left\|P(D)\left[\left(u^{(n)}\right)^{k+1}\right]\right\|_{B_{p, r}^{s-1}} & \leq C\left\|\left(u^{(n)}\right)^{k+1}\right\|_{B_{p, r}^{s-1}} \leq C\left\|u^{(n)}\right\|_{B_{p, r}^{s}}^{k+1} \\
& \leq \frac{C\left\|u_{0}\right\|_{B_{p, r}^{s}}^{k+1}}{\left(1-2 k C\left\|u_{0}\right\|_{B_{p, r}^{s}} t\right)^{\frac{k+1}{k}}} .
\end{aligned}
$$

Consequently,

$$
\left(u^{(n)}\right)_{n} \subset C\left([0, T] ; B_{p, r}^{s}\right) \cap C^{1}\left([0, T] ; B_{p, r}^{s-1}\right) .
$$

Remark Inserting (3.7) into (3.8) yields

$$
\left\|u^{(n)}\right\|_{B_{p, r}^{s}} \leq 4\left\|u_{0}\right\|_{B_{p, r}^{s}}
$$

for $n \in \mathbf{N}$. From (3.11) and (3.7), $\forall n \in \mathbf{N}^{+}$, we have that

$$
e^{C U^{n}} \leq \exp \left[\int_{0}^{t} \frac{C\left\|u_{0}\right\|_{B_{, r}^{s}}^{k}}{1-2 k C\left\|u_{0}\right\|_{B_{p, r}^{s}}^{k} t} d \tau\right] \leq 4
$$

and

$$
\|u(t)\|_{B_{p, r}^{s}} \leq \frac{\left\|u_{0}\right\|_{B_{p, r}^{s}}}{\left(1-2 k C\left\|u_{0}\right\|_{B_{p, r}^{s}}^{k} t\right)^{1 / k}}
$$

with the aid of Fatou's lemma. We define

$$
L=4\left(\left\|u_{0}\right\|_{B_{p, r}^{s}}+1\right)
$$

Thus,

$$
\left\|u^{(n)}\right\|_{B_{p, r}^{s}}+1 \leq L
$$

for $n \in \mathbf{N}^{+}$.

\section{Third step: convergence}

We prove that $\left(u^{(n)}\right)_{n \in \mathbf{N}}$ is a Cauchy sequence in $C\left([0, T] ; B_{p, r}^{s-1}\right)$. For $(m, n) \in \mathbf{N}^{2}$, we have

$$
\begin{aligned}
{\left[\partial_{t}\right.} & \left.+\left(u^{(n+m)}\right)^{k} \partial_{x}\right]\left(u^{(n+1+m)}-u^{(n+1)}\right) \\
= & \left(\left(u^{(n)}\right)^{k}-\left(u^{(n+m)}\right)^{k}\right) \partial_{x} u^{(n+1)} \\
& +Q P(D)\left[\left(u^{(n+m)}-u^{(n)}\right) \sum_{j=0}^{k}\left(u^{(n+m)}\right)^{k-j}\left(u^{(n)}\right)^{j}\right] .
\end{aligned}
$$


Combining (2.3) with (3.20), we have that

$$
\begin{aligned}
& \left\|\left[u^{(n+1+m)}-u^{(n+1)}\right](t)\right\|_{B_{p, r}^{s-1}} \\
& \leq e^{U^{n}(t)}\left(\left\|u_{0}^{(n+1+m)}-u_{0}^{(n+1)}\right\|_{B_{p, r}^{s-1}}\right) \\
& \quad+\int_{0}^{t} e^{U^{n}(t)-U^{n}(\tau)}\left\|F\left(u^{(n)}, u^{(n+m)}, \partial_{x} u^{(n+1)}\right)\right\|_{B_{p, r}^{s-1}} d \tau,
\end{aligned}
$$

where

$$
\begin{aligned}
& U^{n}(t)=\int_{0}^{t}\left\|\left(u^{(n+m)}\right)^{k}\right\|_{B_{p, r}^{s}} d \tau \\
& F\left(u^{(n)}, u^{(n+m)}, \partial_{x} u^{(n+1)}\right) \\
& \quad=\left(\left(u^{(n)}\right)^{k}-\left(u^{(n+m)}\right)^{k}\right) \partial_{x} u^{(n+1)}+Q P(D)\left[\left(u^{(n+m)}-u^{(n)}\right) \sum_{j=0}^{k}\left(u^{(n+m)}\right)^{k-j}\left(u^{(n)}\right)^{j}\right] .
\end{aligned}
$$

By using (3) and (7) of Lemma 2.2, we have that

$$
\begin{aligned}
& \left\|F\left(u^{(n)}, u^{(n+m)}, \partial_{x} u^{(n+1)}\right)\right\|_{B_{p, r}^{s-1}} \\
& \leq C\left\|\left(u^{(n)}\right)^{k}-\left(u^{(n+m)}\right)^{k}\right\|_{B_{p, r}^{s-1}}\left\|\partial_{x} u^{(n+1)}\right\|_{B_{p, r}^{s-1}} \\
& \quad+C\left\|P(D)\left[\left(u^{(n+m)}-u^{(n)}\right) \sum_{j=0}^{k}\left(u^{(n+m)}\right)^{k-j}\left(u^{(n)}\right)^{j}\right]\right\|_{B_{p, r}^{s-1}} \\
& \leq C\left\|u^{(n)}-u^{(n+m)}\right\|_{B_{p, r}^{s-1}}\left[\left\|u^{(n)}\right\|_{B_{p, r}^{s}}+\left\|u^{(n+1)}\right\|_{B_{p, r}^{s}}+\left\|u^{(n+m)}\right\|_{B_{p, r}^{s}}+1\right]^{k} \\
& \leq C L^{k}\left\|u^{(n)}-u^{(n+m)}\right\|_{B_{p, r}^{s-1}} .
\end{aligned}
$$

Inserting (3.22) into (3.21) yields

$$
\begin{aligned}
& \left\|\left[u^{(n+1+m)}-u^{(n+1)}\right](t)\right\|_{B_{p, r}^{s-1}} \\
& \quad \leq e^{U^{n}(t)}\left(\left\|u_{0}^{(n+1+m)}-u_{0}^{(n+1)}\right\|_{B_{p, r}^{s-1}}\right)+C L^{k} \int_{0}^{t} e^{U^{n}(t)-U^{n}(\tau)}\left\|u^{(n)}-u^{(n+m)}\right\|_{B_{p, r}^{s-1}} d \tau .
\end{aligned}
$$

From (3.2) and Lemma 2.1, we can easily obtain that

$$
\left\|u_{0}^{(n+1+m)}-u_{0}^{(n+1)}\right\|_{B_{p, r}^{s-1}} \leq C 2^{-n}
$$

Obviously,

$$
e^{U^{n}(t)} \leq 4, \quad e^{U^{n}(t)-U^{n}(\tau)} \leq 4
$$

Inserting (3.24) and (3.25) into (3.23) leads to

$$
\begin{aligned}
& \left\|\left(u^{(n+1+m)}-u^{(n+1)}\right)(t)\right\|_{B_{p, r}^{s-1}} \\
& \quad \leq C 2^{-n}+C L^{k} \int_{0}^{t}\left\|\left(u^{(n)}-u^{(n+m)}\right)(\tau)\right\|_{B_{p, r}^{s-1}} d \tau .
\end{aligned}
$$


We define

$$
A_{(n, m)}(t)=\left\|\left(u^{(n+m)}-u^{(n)}\right)\right\|_{B_{p, r}^{s-1}} .
$$

Inserting (3.27) into (3.26) leads to

$$
A_{(n+1, m)}(t) \leq C 2^{-n}+C L^{k} \int_{0}^{t} A_{(n, m)}(\tau) d \tau .
$$

We define

$$
\rho_{n}(t)=\sup _{m \in \mathbf{N}^{+}} A_{(n, m)}(t)=\sup _{m \in \mathbf{N}^{+}}\left\|\left(u^{(n+m)}-u^{(n)}\right)(t)\right\|_{B_{p, r}^{s}}
$$

and

$$
\widetilde{\rho}(t)=\limsup _{n \rightarrow+\infty} \rho_{n}(t) .
$$

Combining (3.30) with (3.29), (3.28), by using Fatou's lemma, we have that

$$
\widetilde{\rho}(t)=\limsup _{n \rightarrow+\infty} \rho_{n+1}(t) \leq C^{k} \int_{0}^{t} \widetilde{\rho}(\tau) d \tau .
$$

Applying Gronwall's inequality to (3.31) yields

$$
\widetilde{\rho}(t) \leq e^{t C^{k}} \widetilde{\rho}(0)
$$

for $t \in[0, T]$. According to the definition of $\widetilde{\rho}(t)$, we can easily obtain that

$$
\widetilde{\rho}(0)=0 .
$$

Combining (3.32) with (3.33), we have that

$$
\widetilde{\rho}(t)=0 .
$$

Hence, $\left(u^{n}\right)_{n}$ is a Cauchy sequence in $C\left([0, T] ; B_{p, r}^{s-1}\right)$.

\section{Fourth step: existence in $E_{p, r}^{s}(T)$}

Now we prove that $u \in E_{p, r}^{s}(T)$ and satisfies (1.4)-(1.5) since $\left(u^{n}\right)_{n \in \mathbf{N}}$ is uniformly bounded in $L^{\infty}\left(0, T ; B_{p, r}^{s}\right)$. From (6) in Lemma 2.2 , we have that $u \in L^{\infty}\left(0, T ; B_{p, r}^{s}\right)$. From (1.4), we can easily prove that $u_{t} \in L^{\infty}\left(0, T ; B_{p, r}^{s-1}\right)$. It is easily checked that $u$ is indeed a solution to (1.4)-(1.5) by passing to the limit in (3.1)-(3.2).

Now we prove that $u \in E_{p, r}^{s}(T)$. Since $u \in B_{p, r}^{s}, \forall \epsilon>0$, there exists $q_{0} \in \mathbf{N}^{+}$such that

$$
\sum_{q \geq q_{0}} 2^{q s r}\left\|\Delta_{q} u\right\|_{L^{\infty}\left(0, T ; L^{p}\right)}^{r} \leq \frac{\epsilon}{4}
$$


From the definition of Besov spaces, we have that

$$
\begin{aligned}
& \|u(t+\delta)-u(t)\|_{B_{p, r}^{s}}^{r} \\
& =\sum_{q<q_{0}} 2^{q s r}\left\|\Delta_{q} u(t)-\Delta_{q} u(t+\delta)\right\|_{L^{p}}^{r}+\sum_{q \geq q_{0}} 2^{q s r}\left\|\Delta_{q} u-\Delta_{q} u(t+\delta)\right\|_{L^{p}}^{r} \\
& \leq \sum_{q<q_{0}} 2^{q s r}\left\|\Delta_{q} u(t)-\Delta_{q} u(t+\delta)\right\|_{L^{p}}^{r}+2 \sum_{q \geq q_{0}} 2^{q s r}\left\|\Delta_{q} u(t)\right\|_{L^{\infty}\left(0, T ; L^{p}\right)}^{r} \\
& \leq \sum_{q<q_{0}} 2^{q s r}\left\|\Delta_{q} u(t)-\Delta_{q} u(t+\delta)\right\|_{L^{p}}^{r}+\frac{\epsilon}{2} .
\end{aligned}
$$

By using the mean value theorem, we have that

$$
\left\|\Delta_{q} u(t)-\Delta_{q} u(t+\delta)\right\|_{L^{p}}=\left\|\Delta_{q} u_{t}(t+\theta \delta)\right\|_{L^{p}}|\delta| \leq\left\|\Delta_{q} u_{t}(t)\right\|_{L^{\infty}\left(0, T ; L^{p}\right)}|\delta|,
$$

where $0<\theta<1$. Inserting (3.37) into (3.36) yields

$$
\begin{aligned}
& \|u(t+\delta)-u(t)\|_{B_{p, r}^{s}}^{r} \\
& \quad=\sum_{q<q_{0}} 2^{q s r}\left\|\Delta_{q} u(t)-\Delta_{q} u(t+\delta)\right\|_{L^{p}}^{r}+\sum_{q \geq q_{0}} 2^{q s r}\left\|\Delta_{q} u(t)-\Delta_{q} u(t+\delta)\right\|_{L^{p}}^{r} \\
& \quad \leq|\delta| \sum_{q<q_{0}}\left\|\Delta_{q} u_{t}(t)\right\|_{L^{\infty}\left(0, T ; L^{p}\right)}^{r}+\frac{\epsilon}{2} \\
& \quad \leq|\delta|\left\|u_{t}\right\|_{L^{\infty}\left(0, T ; B_{p, r}^{s-1}\right)}^{r}+\frac{\epsilon}{2} .
\end{aligned}
$$

We may choose $\delta$ sufficiently small such that

$$
|\delta|\left\|u_{t}\right\|_{L^{\infty}\left(0, T ; B_{p, r}^{s-1}\right)}^{r} \leq \frac{\epsilon}{2} .
$$

Inserting (3.39) into (3.38) leads to

$$
\|u(t+\delta)-u(t)\|_{B_{p, r}^{s}} \leq \epsilon^{1 / r} .
$$

Thus, we derive that

$$
u \in C\left([0, T] ; B_{p, r}^{s}\right) .
$$

Combining (3.41) with (1.4), we can easily obtain

$$
u_{t} \in C\left([0, T] ; B_{p, r}^{s-1}\right) .
$$

From (3.41) and (3.42), we have that $u \in E_{p, r}^{s}(T)$.

\section{Fifth step: uniqueness of solution}

The uniqueness of the solution to the Cauchy problem for (1.4) can be proved similarly to Proposition 3.1 of [14]. 
Sixth step: continuity in $E_{p, r}^{s}(T)$ with $s>1+\frac{1}{p}$ (or $s \geq 1+\frac{1}{p}$ if $r=1$ with $p \in[1,+\infty)$ ) The continuity of the solution to the Cauchy problem for (1.4) can be proved similarly to the continuity of the solution to the Degasperis-Procesi equation which can be seen in [14].

\section{Proof of Theorem 1.2}

From (6.5) and (6.7) of [22], we have that

$$
\partial_{x} u_{c}(x, t)=-\operatorname{sign}(x-c t) u_{c}(x, t), \quad \partial_{t} u_{c}(x, t)=c \operatorname{sign}(x-c t) u_{c}(x, t),
$$

where $u_{c}(x, t)=c^{1 / k} e^{-|x-c t|}$. By using integration by parts and (4.1), we have that

$$
\begin{aligned}
& \int_{0}^{+\infty} \int_{\mathbf{R}}\left(u_{c} \partial_{t} \phi+\frac{1}{k+1} u^{k+1} \partial_{x} \phi\right) d x d t+\int_{\mathbf{R}} u_{c}(x, 0) \phi(x, 0) d x \\
& \quad=-\int_{0}^{+\infty} \int_{\mathbf{R}} \phi\left[\partial_{t} u_{c}+u_{c}^{k} \partial_{x} u_{c}\right] d x d t \\
& \quad=-\int_{0}^{+\infty} \int_{\mathbf{R}} \phi \operatorname{sign}(x-c t)\left[c u_{c}-u_{c}^{k+1}\right] d x d t .
\end{aligned}
$$

Since $u_{c}(x, t)=c^{1 / k} e^{-|x-c t|}$, we have that when $x>c t$,

$$
\operatorname{sign}(x-c t)\left[c u_{c}-u_{c}^{k+1}\right]=c^{1+\frac{1}{k}}\left[e^{c t-x}-e^{(k+1)(c t-x)}\right]
$$

and when $x \leq c t$,

$$
\operatorname{sign}(x-c t)\left[c u_{c}-u_{c}^{k+1}\right]=c^{1+\frac{1}{k}}\left[e^{(x-c t)}-e^{(k+1)(x-c t)}\right] .
$$

By using $\left(1-\partial_{x}^{2}\right)^{-1} f=p * f$ and (4.1), we have that

$$
\begin{aligned}
\int_{0}^{+\infty} & \int_{\mathbf{R}} \phi \partial_{x}\left(1-\partial_{x}^{2}\right)^{-1}\left[\frac{k^{2}+2 k}{k+1} u_{c}^{k+1}\right] d x d t \\
& =\int_{0}^{+\infty} \int_{\mathbf{R}} \phi \partial_{x} p *\left[\frac{k^{2}+2 k}{k+1} u_{c}^{k+1}\right] d x d t \\
& =-\frac{k^{2}+2 k}{2(k+1)} c^{1+\frac{1}{k}} \int_{0}^{+\infty} \int_{\mathbf{R}_{x}} \int_{\mathbf{R}_{y}} \phi \operatorname{sign}(x-y) e^{-|x-y|} e^{-(k+1)|y-c t|} d y d x d t .
\end{aligned}
$$

Now, we compute

$$
\begin{aligned}
& -\frac{k^{2}+2 k}{2(k+1)} c^{1+\frac{1}{k}} \int_{\mathbf{R}_{y}} \operatorname{sign}(x-y) e^{-|x-y|} e^{-(k+1)|y-c t|} d y \\
& :=I_{1}+I_{2}+I_{3} .
\end{aligned}
$$


and

$$
\begin{aligned}
I_{2} & =-\frac{k^{2}+2 k}{2(k+1)} c^{1+\frac{1}{k}} \int_{c t}^{x} e^{-(x-y)} e^{-(k+1)(y-c t)} d y \\
& =-\frac{k^{2}+2 k}{2(k+1)} c^{1+\frac{1}{k}} e^{-(x-(k+1) c t)} \int_{c t}^{x} e^{-\frac{k(k+2)}{k+1} y} d y \\
& =-\frac{k+2}{2(k+1)} c^{1+\frac{1}{k}}\left[e^{c t-x}-e^{(k+1)(c t-x)}\right]
\end{aligned}
$$

and

$$
\begin{aligned}
I_{3} & =\frac{k^{2}+2 k}{2(k+1)} c^{1+\frac{1}{k}} \int_{x}^{\infty} e^{(x-y)} e^{-(k+1)(y-c t)} d y \\
& =\frac{k^{2}+2 k}{2(k+1)} c^{1+\frac{1}{k}} e^{(x+(k+1) c t)} \int_{x}^{\infty} e^{-(k+2) y} d y \\
& =\frac{k}{2(k+1)} c^{1+\frac{1}{k}} e^{(k+1)(c t-x)} .
\end{aligned}
$$

Thus, when $x>c t$, from (4.7)-(4.9), we have that

$$
\begin{aligned}
I_{1}+ & I_{2}+I_{3} \\
= & -\frac{k}{2(k+1)} c^{1+\frac{1}{k}} e^{c t-x}-\frac{k+2}{2(k+1)} c^{1+\frac{1}{k}}\left[e^{c t-x}-e^{(k+1)(c t-x)}\right] \\
& +\frac{k}{2(k+1)} c^{1+\frac{1}{k}} e^{(k+1)(c t-x)} \\
= & -c^{1+\frac{1}{k}}\left[e^{(k+1)(c t-x)}-e^{c t-x}\right] .
\end{aligned}
$$

Similarly, when $x<c t$, we can obtain

$$
I_{1}+I_{2}+I_{3}=c^{1+\frac{1}{k}}\left[e^{(k+1)(x-c t)}-e^{x-c t}\right]
$$

From (4.3), (4.4) and (4.10) as well as (4.11), we have that

$$
\begin{aligned}
& \int_{0}^{+\infty} \int_{\mathbf{R}}\left[u_{c} \phi_{t}+\frac{1}{k+1} u_{c}^{k+1} \phi_{x}+p *\left[\frac{k^{2}+2 k}{k+1} u_{c}^{k+1}\right] \phi_{x}\right] d x d t \\
& \quad+\int_{\mathbf{R}} u_{c}(x, 0) \phi(x, 0) d x=0 .
\end{aligned}
$$


Thus, $u_{c}=c^{1 / k} e^{-|x-c t|}$ is the solution in the sense of (4.12). For $c>0$, let $u_{c}(x, t)=c^{1 / k} e^{-|x-c t|}$. Thus $u_{c}(x, t)$ is the solitary wave for (1.1) (or (1.4)).

\section{Proof of Theorem 1.3}

Since when $Q=\frac{k(k+2)}{k+1}$ in (1.4), (1.4) possesses the peaked solitary wave $c^{1 / k} e^{|x-c t|}$, Theorem 1.3 can be proved similarly to Proposition 4 of [19].

\section{Proof of Theorem 1.4}

Since when $Q=\frac{k(k+2)}{k+1}$ in (1.4), (1.4) possesses the peaked solitary wave $c^{1 / k} e^{|x-c t|}$, Theorem 1.4 can be proved similarly to Theorem 3 of [23].

\section{Proof of Theorem 1.5}

In this section, we always assume that $s>1+\frac{1}{p}$ (or $s \geq 1+\frac{1}{p}$ if $r=1$ with $p \in[1,+\infty)$ ).

Proof of Theorem 1.5 Applying $\Delta_{q}$ to (1.4) yields

$$
\left(\partial_{t}+u^{k} \partial_{x}\right) \Delta_{q} u=\left[u^{k}, \Delta_{q}\right] \partial_{x} u+Q P(D) \Delta_{q}\left[\frac{k^{2}+2 k}{k+1} u^{k+1}\right] .
$$

From (2.54) of page 112 in [24], since $s>1+\frac{1}{p}$ (or $s \geq 1+\frac{1}{p}$ if $r=1$ with $p \in[1,+\infty)$ ), we have that

$$
\left\|2^{s q}\right\|\left[u^{k}, \Delta_{q}\right] \partial_{x} u\left\|_{L^{p}}\right\|_{\ell^{r}} \leq C\left\|u_{x}\right\|_{L^{\infty}}^{k}\|u\|_{B_{p, r}^{s}} \leq C\|u\|_{B_{p, r}^{s}}^{k+1} .
$$

By using (4) of Lemma 2.2 and $P(D)$ is an $S^{-1}$-multiplier, since $s>1+\frac{1}{p}$ (or $s \geq 1+\frac{1}{p}$ if $r=1$ with $p \in[1,+\infty))$, we have that

$$
\left\|P(D)\left[\frac{k^{2}+2 k}{k+1} u^{k+1}\right]\right\|_{B_{p, r}^{s}} \leq C\left\|u_{x}\right\|_{L^{\infty}}^{k}\|u\|_{B_{p, r}^{s}} \leq C\|u\|_{B_{p, r}^{s}}^{k+1} .
$$

Going along the lines of the proof of Proposition A.1 of [18], from (7.2) and (7.3), we have that

$$
\begin{aligned}
\|u\|_{B_{p, r}^{s}} & \leq\left\|u_{0}\right\|_{B_{p, r}^{s}}+C \int_{0}^{t}\left\|u_{x}\right\|_{L^{\infty}}^{k}\|u\|_{B_{p, r}^{s}} d \tau \\
& \leq\left\|u_{0}\right\|_{B_{p, r}^{s}}+C \int_{0}^{t}\|u\|_{B_{p, r}^{s}}^{k+1} d \tau .
\end{aligned}
$$

Solving (7.4) yields

$$
\|u\|_{B_{p, r}^{s}} \leq e^{c \int_{0}^{t}\left\|u_{x}\right\|_{L}^{k} d \tau}\left\|u_{0}\right\|_{B_{p, r}^{s}}
$$

Solving (7.5) yields

$$
\|u\|_{B_{p, r}^{s}} \leq \frac{\left\|u_{0}\right\|_{B_{p, r}^{s}}}{\left(1-C k t\left\|u_{0}\right\|_{B_{p, r}^{s}}^{k}\right)^{1 / k}} .
$$


Assume that $T^{\star}$ is the maximal time of existence of the solution to problem (1.4)-(1.5). If $T^{\star}<\infty$, we claim that

$$
\int_{0}^{T^{\star}}\left\|u_{x}\right\|_{L^{\infty}}^{k} d \tau=+\infty
$$

We prove the claim (7.8) by contradiction. If (7.8) is untrue, then from (7.8), we have that

$$
\left\|u\left(T^{\star}\right)\right\|_{B_{p, r}^{s}}<\infty,
$$

which contradicts the fact that $T^{\star}$ is the maximal time of existence of the solution to problem (1.4)-(1.5). Consequently, (7.8) is true. From (7.7), we know that $T^{\star} \geq \frac{1}{C k\left\|u_{0}\right\|_{B_{p, r}^{s}}^{k}}$. Moreover, (7.7) ensures the validity of (3.8).

The proof of Theorem 1.5 is completed.

\section{Competing interests}

We declare that we have no competing interests.

\section{Authors' contributions}

All authors read and approved the final manuscript.

\section{Acknowledgements}

We wish to thank the referee for a careful reading and valuable comments on the original draft. The first author is supported by NNSFC under grant No. 11226185. The third author is supported by Foundation and Frontier of Henan Province under grant Nos. $122300410414,132300410432$.

Received: 12 May 2013 Accepted: 29 August 2013 Published: 08 Nov 2013

\section{References}

1. Degasperis, A, Holm, D, Hone, A: A new integrable equation with peakon solutions. Theor. Math. Phys. 133, 1463-1474 (2002)

2. Lundmark, H: Formation and dynamics of shock waves in the Degasperis-Procesi equation. J. Nonlinear Sci. 17 , 169-198 (2007)

3. Lin, Z, Liu, Y: Stability of peakons for the Degasperis-Procesi equation. Commun. Pure Appl. Math. LXII, 125-146 (2009)

4. Escher, J, Liu, Y, Yin, Z: Global weak solutions and blow-up structure for the Degasperis-Procesi equation. J. Funct. Anal. 241, 457-485 (2006)

5. Liu, Y, Yin, Z: Global existence and blow-up phenomena for the Degasperis-Procesi equation. Commun. Math. Phys. 267, 801-820 (2006)

6. Liu, Y, Yin, Z: On the blow-up phenomena for the Degasperis-Procesi equation. Int. Math. Res. Not. 2007, Article ID rnm117 (2007)

7. Constantin, A, Lannes, D: The hydrodynamical relevance of the Camassa-Holm and Degasperis-Procesi equations. Arch. Ration. Mech. Anal. 192, 165-186 (2007)

8. Henry, D: Infinite propagation speed for the Degasperis-Procesi equation. J. Math. Anal. Appl. 311, 755-759 (2005)

9. Lundmark, H, Szmigielski, J: Multi-peakon solutions of the Degasperis-Procesi equation. Inverse Probl. 19, 1241-1245 (2003)

10. Matsuno, Y: Multisoliton solutions of the Degasperis-Procesi equation and their peakon limit. Inverse Probl. 21, 1553-1570 (2005)

11. Himonas, A, Kenig, CE: Non-uniform dependence on initial data for the $\mathrm{CH}$ equation on the line. Differ. Integral Equ. 22, 201-224 (2009)

12. Himonas, A, Holliman, C: On the well-posedness of the Degasperis-Procesi equation. Discrete Contin. Dyn. Syst. 31, 469-488 (2011)

13. Himonas, A, Misiolek, G, Ponce, G: Non-uniform continuity in $H^{1}$ of the solution map of the CH equation. Asian J. Math. 11, 141-150 (2007)

14. Gui, G, Liu, Y: On the Cauchy problem for the Degasperis-Procesi equation. Q. Appl. Math. LXIX, $445-464$ (2011)

15. Yan, W, Li, Y, Zhang, Y: The Cauchy problem for the integrable Novikov equation. J. Differ. Equ. 253, 298-318 (2012)

16. Chemin, J: Perfect Incompressible Fluids. Oxford Lectures Series in Mathematics and Its Applications, vol. 14. Oxford University Press, New York (1998)

17. Chemin, J: Localization in Fourier space and Navier-Stokes. In: Phase Space Analysis of Partial Differential Equations. CRM Series, pp. 53-136. Scuola Norm. Sup., Pisa (2004)

18. Danchin, R: A few remarks on the Camassa-Holm equation. Differ. Integral Equ. 14, 953-988 (2001)

19. Danchin, R: A note on well-posedness for Camassa-Holm equation. J. Differ. Equ. 192, 429-444 (2003)

20. Danchin, R: Fourier analysis method for PDEs. Lecture notes, 14 November (2005) 
21. Vishik, M: Hydrodynamics in Besov spaces. Arch. Ration. Mech. Anal. 145, 197-214 (1998)

22. Gui, G, Liu, Y, Olver, P, Qu, C: Wave-breaking and peakon for a modified Camassa-Holm equation. Commun. Math Phys. 319, 731-759 (2013)

23. Himonas, A, Misiolek, G: The Cauchy problem for an integrable shallow-water equation. Differ. Integral Equ. 14 831-921 (2001)

24. Bahouri, H, Chemin, J, Danchin, R: Fourier Analysis and Nonlinear Partial Differential Equations. Springer, Berlin (2011)

10.1186/1687-2770-2013-235

Cite this article as: Zuo et al.: The Cauchy problem for the generalized Degasperis-Procesi equation. Boundary Value Problems 2013, 2013:235

Submit your manuscript to a SpringerOpen ${ }^{\circ}$ journal and benefit from:

- Convenient online submission

- Rigorous peer review

Immediate publication on acceptance

- Open access: articles freely available online

- High visibility within the field

- Retaining the copyright to your article

Submit your next manuscript at $>$ springeropen.com 